\title{
Proteomic and bioinformatic analyses of putative Mannheimia haemolytica secretome by liquid chromatography and tandem mass spectrometry
}

\author{
Sahlu Ayalew ${ }^{\mathrm{a}, *}$, Anthony W. Confer ${ }^{\mathrm{a}}$, Steve D. Hartson ${ }^{\mathrm{b}}$, Patricia J. Canaan ${ }^{\mathrm{b}}$, \\ Mark Payton $^{\mathrm{c}}$, Brian Couger ${ }^{\mathrm{d}}$ \\ a Department of Veterinary Pathobiology, Center for Veterinary Health Sciences, Oklahoma State University, Stillwater, OK 74078, USA \\ b Department Biochemistry and Molecular Biology, Oklahoma State University, Stillwater, OK 74078, USA \\ ${ }^{\mathrm{c}}$ Department of Statistics, Oklahoma State University, Stillwater, OK 74078, USA \\ ${ }^{\mathrm{d}}$ High Performance Computing Center, Oklahoma State University, Stillwater, OK 74078, USA
}

\section{A R T I C L E I N F O}

\section{Article history:}

Received 19 July 2016

Received in revised form 17 January 2017

Accepted 22 February 2017

\section{Keywords:}

Mannheimia haemolytica

Secretome

Secretory peptides

PSORTb

SignalP 4.1

SecretomeP

LipoP

TatP

Phobius SP and PRED-TAT

LC-MS/MS

\begin{abstract}
A B S T R A C T
Mannheimia haemolytica is a major bacterial contributor to bovine respiratory disease complex that costs the livestock industry a billion dollars a year in USA. Commercial vaccines are only partially efficacious under field conditions. Earlier studies found that outer membrane protein preparations and culture supernatants can induce immune responses that enhance resistance to challenge by M. haemolytica strains. The objective of this study was to characterize secretome of two M. haemolytica stains grown under two different media. Bacteria-free concentrated supernatants from M. haemolytica culture was subjected to LC-MS/MS. The secretome of M. haemolytica from both strains yielded 923 proteins. Using bioinformatic tools, 283 were identified as secreted proteins. Further breakdown of 283 proteins showed that $114(40.2 \%), 184(65.0 \%), 138(48.7 \%), 151$ (53.3\%) and $172(60.7 \%)$ were characterized as secreted proteins by SignalP 4.1, SecretomeP 2.0, LipoP, Phobius, and PRED-TAT, respectively. A total of 95 (33.56\%) proteins were characterized as being secreted via non-classical pathway as opposed to the majority that were secreted in signal peptide dependent pathway. The demonstrated proteins include all previously immunologically characterized $M$. haemolytica proteins. The potential of using secretome analysis in the design and development of a multivalent vaccine is discussed.
\end{abstract}

(c) 2017 Elsevier B.V. All rights reserved.

\section{Introduction}

Bovine respiratory disease (BRD) is the major cause of economic losses to the beef cattle industry (Miles, 2009). In feedlot cattle, Mannheimia haemolytica (formerly Pasteurella haemolytica) serotype 1 is most commonly associated with bovine pneumonia found in feedlot cattle known as Shipping Fever (Frank, 1986, 1989). Much effort has been expended toward development and improvement of vaccines for prevention of Shipping Fever (Confer, 1993). Current vaccines are largely ineffective or of limited efficacy under field conditions in a feedlot (Larson and Step, 2012; Perino and Hunsaker, 1997). The development of more efficacious vaccines will require a thorough understanding of virulence factors, the outer membrane proteins (OMPs), and secreted proteins of $M$. haemolytica as well as the host immune response to these

\footnotetext{
* Corresponding author.

E-mail address: sahlu.ayalew@okstate.edu (S. Ayalew).
}

factors. Such a vaccine will most likely contain antigenic components derived through recombinant DNA technology.

Shewen and Wilkie (1988) demonstrated that immunity against $M$. haemolytica requires leukotoxin (LKT) neutralizing antibodies as well as antibodies against bacterial cell surface antigens. Studies in our and others' laboratories demonstrated that high antibody responses to OMPs, ranging from 16 to $86 \mathrm{kDa}$, correlated with resistance to challenge with virulent $M$. haemolytica S1, and vaccination of cattle with OMP-enriched cellular fractions from $M$. haemolytica S1 enhanced resistance of cattle against experimental challenge (Ayalew et al., 2010, 2011a,b; Confer et al., 2003, 2006; Morton et al., 1995; Mosier et al., 1989; Orouji et al., 2012). In this regard, we used immunoproteomics, which uses a combination of 2D-gel electrophoresis (2-DE) and Western blotting with bovine convalescent sera, and identified 132 immunoreactive proteins in outer membrane preparations of M. haemolytica using LC-MS/MS. Of those, 55 are putative vaccine candidates (Ayalew et al., 2010). 
Demonstration of the importance of neutralizing antibodies to the $105 \mathrm{kDa}$ LKT led to the development of a commercial culture supernatant-based vaccine, and preliminary examination of secreted $M$. haemolytica immunogens that are of potential importance (Gentry et al., 1985; Mosier et al., 1994; Shewen and Wilkie, 1988). Mosier et al. (1994) demonstrated that antibodies to five proteins in culture supernatant ranging from 16 to $100 \mathrm{kDa}$ correlated with resistance to experimental challenge. In addition, a $32.5 \mathrm{kDa}$ sialoglycoprotease was identified in $M$. haemolytica supernatants and antibodies to that protein correlated with resistance (Lee et al., 1994).

Because LKT is secreted by M. haemolytica into the culture supernatant as well as various bacterial enzymes and proteins of unknown origin, we undertook to examine the proteins within the culture supernatant (secretome) and determine which are potentially secreted proteins. Gram-negative bacteria have developed several pathways for secreting cellular components into their immediate environments for several purposes (Costa et al., 2015; Green and Mecsas, 2016; Lycklama and Driessen, 2012; Palmer and Berks, 2012). These highly sophisticated and dedicated protein secretion systems fall into at least eight characterized secretion systems (Sec, Tat, T1SS, T2SS, T3SS, T4SS, T5SS and T6SS) that either work in tandem or individually to effect the transport of substrates across inner and outer membrane into the external environment (Costa et al., 2015; Green and Mecsas, 2016; Lycklama and Driessen, 2012; Palmer and Berks, 2012). In addition to these dedicated secretion systems, Gram-negative bacteria also utilize outer membrane vesicles to export cellular components (Kuehn and Kesty, 2005). Such vesicles have been shown to carry proteins, DNA, RNA, lipids, etc., originating from the cells from which vesicles slough off. With the exception of vesicles, whose mechanism of formation has not been clearly established, dedicated secretion systems identify which protein is secreted by what mechanism by the nature of secretion signals, also referred to as signal peptides (Green and Mecsas, 2016). Bioinformatic protein prediction tools use the presence or absence of signal peptides, their locations ( $\mathrm{N}$ or $\mathrm{C}$ termini), amino acid composition, and other criteria to predict if and how bacteria secrete proteins. We present here that in many cases none of the bioinformatic tools used can conclusively predict or completely agree if a protein is a secreted protein. The objective of this research was to study the secretomes of two virulent $M$. haemolytica strains, characterize the proteins as to whether they are potentially secreted proteins, and identify if proteins that have been shown to be highly immunogenic are present in the secretome. To accomplish this objective, bacteria-free concentrated supernatants from $M$. haemolytica cultures were subjected to trypsinolysis and LC-MS/MS.

\section{Materials and methods}

\subsection{M. haemolytica strains and culture}

Two virulent strains of $M$. haemolytica were used in this study. While both belong to serotype 1 (S1), strain $89010807 \mathrm{~N}$ was isolated in 1989 and Oklahoma (OKL) was isolated in 1978. Both were isolated from lungs of feedlot calves with pneumonia (Pandher et al., 1999). We used these two strains with consistent disease causing profile in our hands to show the reproducibility of secretome production and if passage in our laboratories have in anyway impacted the same. Supernatants from three biological replicates (same strain grown in three flasks with the same medium) of each strain grown in two media, a total of 12 biological replicates, were prepared for studying secretome profiles. Two growth media viz., Brain Heart Infusion (BHI), a complex and inexpensive medium routinely used in culturing this and other fastidious bacteria and RPMI-1640, a synthetic medium, routinely used to grow this organism albeit expensive were used in this study. To achieve this, well isolated colonies of $M$. haemolytica strains from BHI agar plates supplemented with $5 \%$ defibrinated sheep blood were transferred into triplicates of culture tubes containing BHI broth and incubated overnight in a shaker incubator. The overnight starter cultures were transferred (1/100th volume) into 3 Erlenmeyer flasks each containing $250 \mathrm{ml}$ of RPMI-1640 medium and an additional 3 flasks containing $250 \mathrm{ml}$ of BHI. Both sets of cultures were incubated in an orbital shaker ( $150 \mathrm{rpm}$ ) at $37^{\circ} \mathrm{C}$. Growth was stopped at late log phase by centrifugation of each culture at $12,000 \times \mathrm{g}$ for 20 minutes and pellets were discarded. Supernatants from each were filtered through $0.2 \mu \mathrm{M}$ Nalgene filters (Thermo Scientific, Waltham, MA) to remove any bacterial cells and concentrated with Centricon Plus-70 Centrifugal filter devices with 5000 Da MWCO (Millipore, Billerica, MA). Protein concentration in each preparation was determined by Bicinchoninic acid (BCA) assay. The preparations were submitted to the Recombinant DNA/Protein Resource Facility, Oklahoma State University, for identification using LC-MS/MS as described below. Three technical replicates of concentrated BHI was analyzed in the same manner to identify proteins contributed by the medium.

\subsection{LC-MS/MS methodology}

Proteomes from 12 biological and 3 technical samples were analyzed as described previously (Voruganti et al., 2013), but using chromatography columns packed with $40 \mathrm{~cm}$ of 3-micron Magic C18 AQ particles (Bruker) and eluted using a $2.8-44 \%$ acetonitrile gradient over a period of $262 \mathrm{~min}$. LC-MS/MS RAW files were analyzed with MaxQuant v1.5.3.8 (Cox and Mann, 2008), using 1\% protein and peptide FDR thresholds to search a database of 15,043 Mannheimia sequences downloaded from Uniprot on 07/16/15 (see Supplementary Table for the full description of the MaxQuant settings used). Relative abundance of proteins was determined on the basis of their normalized peptide intensities via the Label Free Quantification (LFQ) algorithm in MaxQuant v1.5.3.8 (Cox et al., 2014; Cox and Mann, 2008). Within the MaxQuant software output, this parameter is referred to as LFQ intensity.

\subsection{Bioinformatic analysis}

To maximize the accuracy of peptide identification through mass spectrometry, multiple bioinformatics algorithms (Romine, 2011) for prediction of protein secretion were leveraged which make use of different prediction methodology and account for non-canonical secretion. The prediction softwares included and brief descriptions are as follows. Amino acid sequences of identified proteins in FASTA formats were analyzed using bioinformatic tools shown here. PSORTb (http://www.psort.org/ psortb), predicts subcellular locales of bacterial proteins (Yu et al., 2010). SignalP 4.1 (http://www.cbs.dtu.dk/services/SignalP) predicts the presence and location of signal peptide cleavage sites (Petersen et al., 2011). SecP (http://www.cbs.dtu.dk/services/ SecretomeP) is a bacterial non-classical secretion and a prediction method for identification of proteins following signal peptide independent secretion pathways (Bendtsen et al., 2005a). LipoP (http://www.cbs.dtu.dk/services/LipoP/) generates predictions of lipoproteins and discriminates between lipoprotein signal peptides, other signal peptides and n-terminal membrane helices in Gram-negative bacteria (Juncker et al., 2003), TatP (http://www. cbs.dtu.dk/services/TatP) predicts the presence and location of Twin-arginine signal peptide cleavage sites in bacteria (Bendtsen et al., 2005b), and PRED-TAT (http://www.compgen.org/tools/ PRED-TAT), a fairly recent addition to the toolbox of bioinformatics, 
is capable of distinguishing Sec from Tat signal peptides and predicting their cleavage sites (Bagos et al., 2010). Phobius (http://phobius.cgb.ki.se and http://phobius.binf.ku.dk), a combined transmembrane topology and signal peptide predictor was also used (Kall et al., 2007).

All genes identified with proteomics were analyzed for prediction of secretion with each program. Functional annotation of all proteins was achieved using a combination of homology comparisons with NCBI Blastp (Camacho et al., 2009), protein domain prediction (Eddy, 2011) using hmmscan (Finn et al., 2015) against the PFAM 27.0 database (Finn et al., 2014), KOG categories (Galperin et al., 2015; Tatusov et al., 1997) and enzyme activity using the Uniprot (UniProt, 2015) relational database. Genes were established as putative virulence factors that had high homology to established virulence factors or contained information in the Uniprot database that suggested the gene was related to virulence. In addition, all genes that were most likely of intracellular, such as high copy number genes and ribosomal proteins, through there functional category were marked for their location in the secretome comparison between conditions. For all proteins, which had an ambiguous annotation such as "hypothetical" or "predicted protein," each were manually annotated against the Uniprot database and searched against other databases such as NCBI and Brenda (Chang et al., 2015).

\subsection{Statistical analysis}

All analyses were conducted in SAS Version 9.4 (SAS Institute, Cary, NC). The count response was transformed using a log base 2 function in order to alleviate heterogeneous variances typically associated with count data. Mixed model analysis of variance methods were conducted assuming a two-factor treatment structure (strain and medium). Simple effects of strain given medium and medium given strain were calculated and tested using protected pairwise comparisons. This analysis was conducted for each protein. A significance level of 0.05 was used to determine statistical significance.

\section{Results and discussion}

\subsection{General findings}

A total of 923 proteins were identified in supernatants of two $M$. haemolytica stains grown in BHI and RPMI-1640. The complete list of protein groups and their distribution among the two strains and media is given in Supplementary Table and Fig. 1. Overall, there

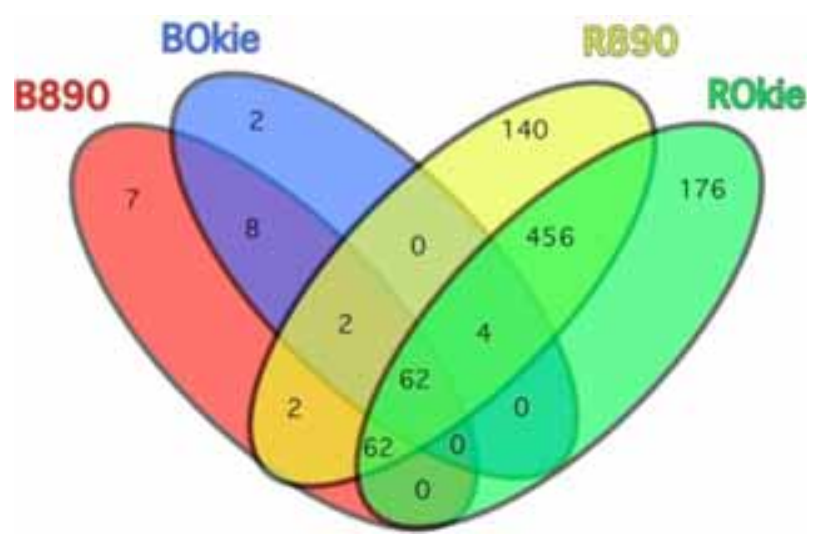

Fig. 1. An overlap of proteins identified in M. haemolytica $89010807 \mathrm{~N}$ and Okie strains grown in two media. B890 and BOkie are proteins identified in supernatants of the two strains, here shown as 890 and Okie, grown in BHI. R890 and ROkie are results from strains cultured in RPMI-1640. were significantly $(p<0.05)$ more proteins identified in RPMI-1640 (584.7) than in BHI (69). There were no significant $(p>0.05)$ differences in the number of proteins identified in BHI supernatants of $M$. haemolytica $89010807 \mathrm{~N}$ and OKL strains. Similarly, the numbers of proteins identified in supernatants of both strains grown in RPMI-1640 were not significantly different $(p>0.05)$. However, there were significant differences between numbers of proteins identified in supernatants of M. haemolytica $89010807 \mathrm{~N}$ grown in BHI and RPMI-1640 and the same is true for OKL strains grown in the two media. A complete list is given in Supplementary Table.

In addition to comparison of the number of secreted proteins, peptide counts were used to compare performances of strains in the two growth media. Statistical analysis of peptide data shows that there were significant differences between strains grown in BHI $(p<0.05)$ whereas the opposite is true when the two strains were grown in RPMI-1640 (Table 1 ). However, for each strain, there were significant differences $(p<0.05)$ of peptide counts in BHI and RPMI-1640 (Table 2). Moreover, responses of both strains in the two media were tested by analyzing MS/MS (spectral counts) data. Analysis of variance was performed on the log2-transformed data, and $p$-values reflect the analysis based on those transformed values. Analysis of a $2 \times 2$ factorial arrangement of treatments, and simple effects are compared (Tables 3 and 4). MS/MS counts show that generally strains perform better in RPMI-1640 as opposed to BHI (Tables 3 and 4). Given medium, 89010807N has significantly higher spectral count than OKL strain, where as there are no significant differences in performances of both strains in RPMI1640 (Table 3). As is alluded to earlier, for each strain, RPMI-1640 is significantly superior to BHI using MS/MS counts as measures (Table 4).

The composition of secretomes in supernatants of two strains grown in the two culture media were also analyzed using LFQ intensities of individual proteins across all treatments. In this instance, only proteins that exhibited LFQ readings in at least two of three biological replicates were included. Proteins with LFQ intensity in only one of three biological replicates were excluded. This resulted in the analysis of a subset of the total proteins, 923. Consequently, in supernatants from cultures in BHI, only 48 proteins were included in this analysis (Suppl. Table I). For the 48 proteins, only 3 showed significant differences in LFQ intensities $(p<0.05)$, wherein one of the proteins exhibited a 2.5 fold LFQ intensity in supernatants from $89010807 \mathrm{~N}$, and the other two had 2.2 and 1.3 fold intensities in OKL. Even though analysis of the data shows significant differences were observed in only three proteins, fold increase analysis of the remaining 45 proteins shows that substantially more proteins are recovered from supernatants of strain $89010807 \mathrm{~N}$ than strain OKL grown in BHI.

When both strains were grown in RPMI-1640, substantially more proteins (493), have met the criterion stated in the previous section and were analyzed using intensities of each protein (Supplementary Table). Of 493 proteins, 77 had significant differences $(p<0.05)$ in LFQ intensities. Analysis of fold increase of all 493 proteins shows that 37 and 33 proteins in supernatants of

Table 1

Peptide analysis. Comparisons of peptide counts of two strains grown in the same medium.

\begin{tabular}{llllc}
\hline Medium & Strain & MNRESP & SERESP & $p$-value \\
\hline $\mathrm{B}$ & 890 & 0.79487 & 0.06972 & $<0.0001$ \\
$\mathrm{~B}$ & OKL & 0.61322 & 0.06849 & \\
$\mathrm{R}$ & 890 & 5.26761 & 0.13669 & 0.6667 \\
$\mathrm{R}$ & OKL & 5.31239 & 0.13545 & \\
\hline
\end{tabular}

B - BHI; R - RPMI-1640; MNRESP - mean response; SERESP - standard error; 890 M. haemolytica $89010807 \mathrm{~N}$ and OKL- M. haemolytica OKL strain. 
Table 2

Peptide analysis. Comparisons of peptide counts of a strain grown in two media.

\begin{tabular}{lllll}
\hline Strain & Medium & MNRESP & SERESP & $p$-value \\
\hline 890 & $\mathrm{~B}$ & 0.79487 & 0.06972 & $<0.0001$ \\
890 & $\mathrm{R}$ & 5.26761 & 0.13669 & \\
OKL & $\mathrm{B}$ & 0.61322 & 0.06849 & $<0.0001$ \\
OKL & $\mathrm{R}$ & 5.31239 & 0.13545 & \\
\hline
\end{tabular}

Table 4

Comparisons of Log2 spectral (MS/MS) counts when each strain is grown in two media.

\begin{tabular}{llrll}
\hline Strain & Medium & MNRESP & SERESP & $p$-value \\
\hline 890 & $\mathrm{~B}$ & 1.2857 & 0.13918 & $<0.0001$ \\
890 & $\mathrm{R}$ & 10.0621 & 0.32743 & \\
OKL & $\mathrm{B}$ & 0.9866 & 0.13882 & $<0.0001$ \\
OKL & $\mathrm{R}$ & 10.1795 & 0.31864 & \\
\hline
\end{tabular}

B - BHI; R - RPMI-1640; MNRESP - mean response; SERESP - standard error; 890 M. haemolytica $89010807 \mathrm{~N}$ and $\mathrm{OKL}-M$. haemolytica OKL strain.

89010807N and OKL, respectively, have at least 1.5 times the intensities.

Comparative analysis based on intensities of individual proteins was also done when a given strain was grown in BHI and RPMI1640. Details are provided in Supplementary Table. Accordingly, when $89010807 \mathrm{~N}$ was grown in BHI or RPMI-1640, 55 proteins were identified of which 28 (50.9\%) exhibited significant differences in intensities $(p<0.05)$. Similarly, 49 proteins that meet the criterion stated above were identified when OKL was grown in the two media of which 31 (63.2\%) had significant differences in LFQ intensities.

\subsection{Bioinformatic analyses}

Whether the 923 proteins identified in this study are truly secreted or the outcome of leakage of proteins due to cell lysis was examined by subjecting the amino acid sequence of each protein to bioinformatics tools that predict secreted proteins using different algorithms. 283 of the 923 proteins were identified or predicted to be secreted proteins by at least one bioinformatics program
Table 3

Comparisons of Log2 spectral (MS/MS) counts when two strains are grown in the same medium.

\begin{tabular}{lllll}
\hline Medium & Strain & MNRESP & SERESP & $p$-value \\
\hline B & 890 & 1.2857 & 0.13918 & $<0.0001$ \\
B & OKL & 0.9866 & 0.13882 & \\
R & 890 & 10.0621 & 0.32743 & 0.9832 \\
R & OKL & 10.1795 & 0.31864 & \\
\hline
\end{tabular}

B - BHI; R - RPMI-1640; MNRESP - mean response; SERESP - standard error; 890 M. haemolytica $89010807 \mathrm{~N}$ and OKL - M. haemolytica OKL strain.

(Supplementary Data). One of the bioinformatic tools that may not necessarily predict secretion but rather subcellular locales and hence indirectly suggest secretion is PSORTb. According to PSORTb, out of 283 putatively secreted proteins, 26 (9.2\%) are outer membrane proteins, 56 (19.79\%) periplasmic, 7 (2.47\%) extracellular, 29 (10.25\%) cytoplasmic membrane, 77 (27.21\%) cytoplasmic and $88(31.10 \%)$ are unknown (Fig. 2). Among the 6 groups of proteins shown in Fig. 2, at least three of them i.e., periplasmic, outer membrane or extracellular, can tentatively qualify as secreted proteins, because each protein must traverse either inner membrane or both inner and outer membranes. It does not, however, mean that proteins designated as cytoplasmic or unknown in Fig. 2 are not secreted. Further analysis using other bioinformatic tools shows that some of them are predicted to be secreted while a few in the three tentatively secreted groups are not.

The 283 putative secreted proteins were subjected to four bioinformatic tools (SignalP4.1, Secretome P, Phobius and PREDTAT) that use dedicated algorithms to predict if proteins are secreted. The most common protein export methods in Gramnegative bacteria depend on systems that recognize signal peptides. The numbers of proteins predicted as possessing the required signal peptide and, therefore, qualify as classically secreted proteins varied among the bioinformatic tool used (Table 5).

Comparison of the four tools shows that Secretome P with 184 $(65.0 \%)$ has identified the largest number of proteins as secreted proteins and SignalP4.1 with only $114(40.2 \%)$ has identified the least. More proteins were identified by Secretome P probably because this tool not only identifies proteins with signal peptides indicating secretion by classical methods but also identifies non-

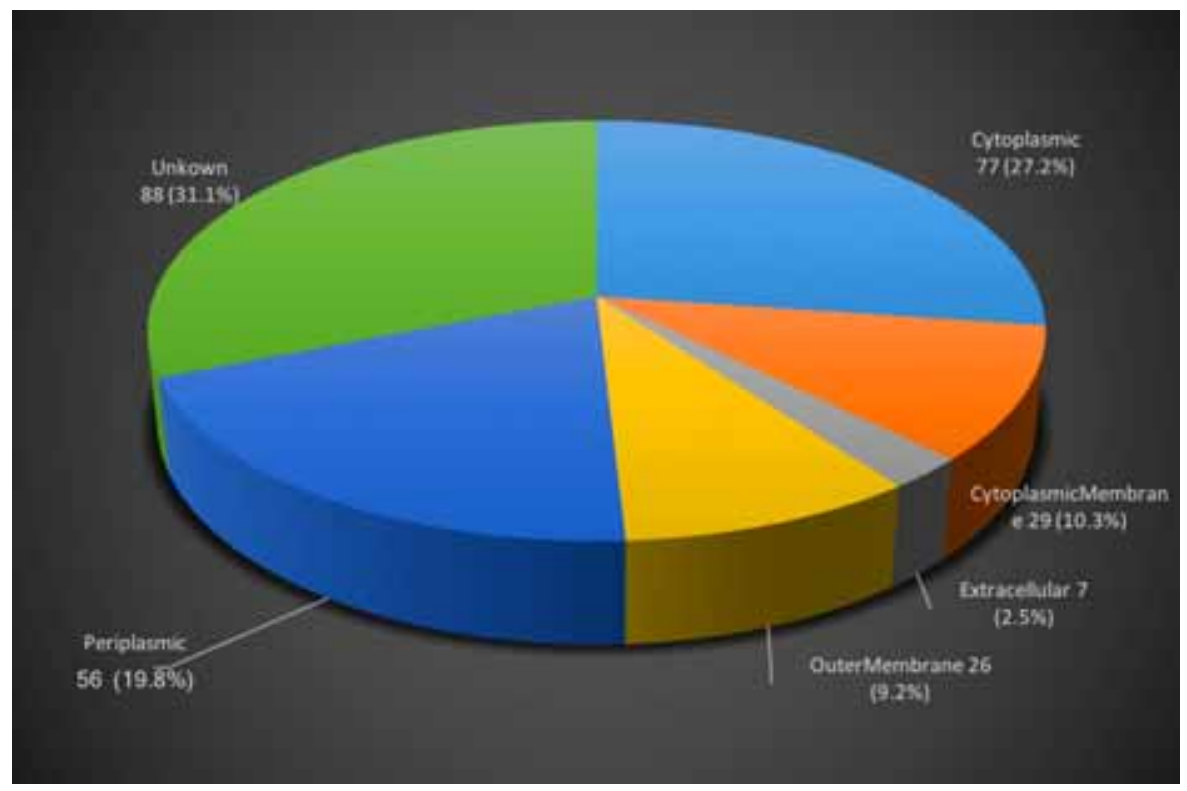

Fig. 2. Pie chart with numbers and percentages of subcellular locales of putative secreted proteins identified in $M$. haemolytica secretome. 
Table 5

Predictions of putative secreted proteins using five bioinformatic tools.

\begin{tabular}{llllll}
\hline Tool & SignalP4.1 & $\begin{array}{l}\text { Secretome P } \\
2.0\end{array}$ & Phobius & PRED-TAT & LipoP \\
& & & & \\
\hline & $114(40.2 \%)$ & $184(65.0 \%)$ & $151(53.3 \%)$ & $172(60.7 \%)$ & $138(48.76 \%)$ \\
\hline
\end{tabular}

A total of 283 proteins were predicted to be secreted proteins in M. haemolytica secretome. This table shows actual numbers and percentages in parenthesis identified by each bioinformatic tool as meeting the criterion set by each algorithm.

classical secretion methods. To separate non-classically from classically secreted proteins, the 184 proteins were subjected to SignalP4.1 to identify proteins with or without signal peptides. Accordingly, out of 184 proteins whose SecP score was $>0.5,89$ (31.5\%) were predicted to have SignalP4.1 score of $>0.51$, confirming the presence of signal peptides. Therefore, 89 proteins are predicted to be secreted by the classical protein secretion pathway, whereas the remaining 95 proteins (33.56\%) are potentially secreted by non-classical pathways.

In the proceeding section, attempts were made to show how SecretomeP can be used to predict secreted proteins and in conjunction with SignalP 4.1 can be used to determine whether the secretory pathway is signal peptide dependent (classic) or signal peptide independent and hence non-classic pathway. It is evident from Table 5 that 114 (12.35\%) of all 923 proteins identified in this study are characterized as having signal peptide. A comparison of SignalP4.1 and SecretomeP as predictors of classically secreted proteins, the former with $114(40.28 \%)$ has identified more signal peptide-dependent secreted proteins out of 283 putative secreted proteins, than the latter 89 (31.44\%).

TatP is a bioinformatic tool that identifies proteins exported by twin-arginine translocase (Tat) secretion system. TatP predicts proteins that have twin-arginine (Tat) signal peptides. Such proteins are exported into the periplasmic compartment or extracellular environment independent of the classical Secdependent translocation pathway (Bagos et al., 2010). The twinarginine translocase (Tat) was described as recognizing longer and less hydrophobic signal peptides carrying a distinctive pattern of two consecutive Arginine (RR) in the N-region (Bagos et al., 2010). Proteins are assigned TatP scores ranging between 0 and 1 and the cutoff to qualify for being secreted via this pathway is 0.36 . In this study, only 20 proteins meet this requirement and this is $2.16 \%$ of the 923 proteins identified in this study, a percentage that is observed in all of Gram-negative bacteria. TatP is more common in Archaea and rare in Gram-negative bacteria and the finding in this study confirms the latter.

PRED-TAT (Petersen et al., 2011), a bioinformatic tool that predicts both Sec and Tat signal peptides, distinguishes between the two signal peptides, and predicts cleavage sites for both (Petersen et al., 2011) was also used. 172 (18.6\%) of 923 proteins characterized in this study have either a Sec (161) or Tat (7) signal peptide (Table 5 ). In addition, four proteins with transmembrane regions were identified (data not shown). A comparison of proteins with Sec signal peptide to other tools reveals that there are less than $45 \%$ agreements in their prediction.

Another tool, LipoP, which predicts lipoprotein signal peptides in Gram-negative bacteria, was used to further characterize proteins identified in this study. According to Juncker et al., this method distinguishes between lipoproteins cleaved by signal peptidase II (SpII proteins), SpI cleaved proteins, cytoplasmic proteins and transmembrane proteins (Juncker et al., 2003). Analysis sf 283 putative secreted proteins by LipoP shows that 145 were identified as cytoplasmic, 106 as SpI and 32 as SpII proteins. According to Juncker et al., these lipoprotein signal peptides are quite similar to the signal peptides of secreted proteins (Juncker et al., 2003). The latter is confirmed by this finding because of the 106 SpI proteins, 84 (79.2\%) had signal peptides according to SignalP 4.1 and 71 (67.00\%) had SecP scores of $>0.5$.

Finally, Phobius (http://phobius.cgb.ki.se and http://phobius. binf.ku.dk) (Kall et al., 2007) was used. According to this predictor 151 (53.35\%) out of 283 proteins have signal peptide and none exhibited Trans Membrane Helices (TMH). Out of 151 proteins with signal peptides, 106 have SecP scores $>0.5,135$ are either SpI or SpII according to LipoP and 114 have SignalP4.1 >0.51. At the same time, five proteins with TMH but no signal peptide have been identified by this bioinformatic tool.

\subsection{Comparisons of experimental and genomic data}

Amino acid sequences of posted genomic data at the time of analysis of Mannheimia haemolytica (5365), M. haemolytica USDA ARS MARC 183 (5267) and M. haemolytica M42548 (5655) protein entries shown in parenthesis were subjected to two of bioinformatic tools to compare and determine similarities and discrepancies between the experimentally generated secretome data in this study (923 proteins) and posted genomic sequences. Deduced amino acid sequences of all proteins of the three data sets, and findings were analyzed using SecretomeP 2.0 and SignalP 4.1 (Table 6).

There are substantially more proteins identified by SecretomeP 2.0 and SignalP 4.1 in the posted genomic data as opposed to experimental data. The relatively higher number of potentially secreted proteins in the genomic data is because the latter depicts theoretical information as opposed to experimental data. It is also worth noting that there are differences between proteins potentially being secreted and actually being secreted. Moreover, not all secreted proteins with all characteristic features of being secreted will do so at the same time. The nature, quantity of secreted proteins are dictated by environmental and growth factors. This probably explains the experimental results obtained in this study. Growth of M. haemolytica in the media and environment in which it is grown was not conducive enough for many of potentially secreted proteins to have been identified in this study. Undoubtedly, environmental queues necessary for some proteins to be secreted were not present in these experimental conditions. Secreted proteins are synthesized and released into the immediate environment in response to myriads of environmental

Table 6

Comparison of experimental data to published genomic sequences.

\begin{tabular}{|c|c|c|c|c|}
\hline Bioinformatic tools & This study & Mannheimia haemolytica & Mh M42548 & Mh USDA, ARS, MARC 183 \\
\hline SecretomeP 2.0 & $184(19.9 \%)$ & $939(17.5 \%)$ & $1001(17.7 \%)$ & $885(16.8 \%)$ \\
\hline SignalP 4.1 & $114(12.35 \%)$ & $474(8.8 \%)$ & $482(8.7 \%)$ & $430(8.2 \%)$ \\
\hline Non-classically secreted proteins & $95(10.29 \%)$ & $603(11.3 \%)$ & $688(12.2 \%)$ & $582(11.1 \%)$ \\
\hline Outer membrane proteins & $26(2.8 \%)$ & $108(2.2 \%)$ & $112(2.0 \%)$ & $116(2.2 \%)$ \\
\hline Extracellular & $7(0.75 \%)$ & $37(0.6 \%)$ & $40(0.7 \%)$ & $35(0.7 \%)$ \\
\hline Periplasmic & $56(6.1 \%)$ & $150(2.8 \%)$ & $156(2.7 \%)$ & $183(3.5)$ \\
\hline
\end{tabular}

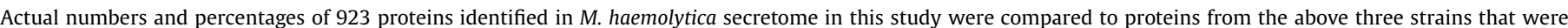
characterized as shown above by SecretomeP 2.0, SignalP 4.1 and PSORTb. 
stimuli and simply growing in any of the two media may not have created the appropriate conditions for the proteins to be released.

There are substantial similarities both in numbers and percentages of different groups of putatively secreted proteins between experimental data and that inferred from posted genomes of $M$. haemolytica. In the present study, SecretomeP 2.0 identified $19.9 \%$ of the proteins as secreted, compared to $16.8 \%$ to $17.7 \%$ for the other three data sets. Similarly, SignalP 4.1 characterized $12.35 \%$ of the proteins in this study as having signal peptides, whereas in the other three $M$. haemolytica sequences lower percentages (8.2-8.7\%) were identified as having signal peptides. Relative abundance of proteins predicted to be outer membrane proteins in the genomic data ranged between 2.0 and $2.7 \%$ and those in this study is 9.18\%. Likewise, proteins characterized as periplasmic (6.1\%) by PSORTb are elevated in this study compared to those in the genomic data (2.7-3.5\%). However, proteins identified as extracellular in all groups are almost equally represented in both experimental and posted genomic data (see Table 6). A deeper analysis of the 7 proteins characterized as extracellular shows that 4 do not form signal peptide commonly seen in Sec-dependent secretion pathways. Two of the 4, however, were predicted to have tatP signal peptide, a kind of signal peptide commonly seen in Archaea and the remaining 2 are secreted by non-classic pathway. More over proteins with SecP score of $>0.5$ and SignalP4.1 score of $<0.51$ are characterized as non-classically secreted proteins. Leukotoxin A is a classic example of non-classically secreted protein. It is predicted to be cytoplasmic by LipoP, has SecP score of 0.854 and SignalP4.1 score of 0.272 thus fulfilling all of the criteria of such proteins. The remaining three are secreted by classic sec-dependent pathway.

Generally, 283 proteins were characterized as potentially secreted proteins by meeting the criteria in at least one of the bioinformatics programs used in this study. Similarly, approximately 885-1001 have been characterized to be so when deduced amino acid sequences of genomes were subjected to the same bioinformatics tools. With $30.6 \%$, close to twice as much proteins in percentage have been identified as secreted proteins compared to $17.5 \%$ proteins of those posted genomes. It is not surprising that relatively more proteins are identified in this study because these proteins were identified in culture supernatants.

\subsection{Secretomes as multivalent vaccines}

The objective of this work is to identify proteins in culture supernatants (secretomes) of two M. haemolytica strains in two growth media commonly used to grow this bacterium with the hope of characterizing potentially immunogenic proteins for use in $M$. haemolytica vaccines. It has been established that bacteria express surface and secreted proteins (the secretome) contain a large number of proteins which they in turn utilize to interact with other microbes, host and/or environment (Gagic et al., 2016). Secretomes in culture supernatants of bacteria have also been used as sources for the identification of proteins involved in virulence (Konecna et al., 2010; Rice et al., 2007; Rodriguez-Alvarez et al.,

Table 7

Some proteins in $M$. haemolytica secretome that have been identified as immunogens in previous studies.

\begin{tabular}{|c|c|c|c|c|c|c|c|c|c|}
\hline \multirow[t]{2}{*}{ Protein } & \multirow{2}{*}{$\begin{array}{l}\text { M. haemolytica } \\
\text { Strains }\end{array}$} & \multicolumn{3}{|c|}{ Relative abundance } & \multicolumn{5}{|c|}{ Bioinformatics prediction of secreted protein } \\
\hline & & $\mathrm{BHI}$ & RPMI & Fold & SignalP4.1 & SecretomeP 2.0 & Phobius & PRED-TAT & LipoP \\
\hline \multirow[t]{2}{*}{ Leukotoxin } & $89010807 \mathrm{~N}$ & $5.10 \mathrm{E}+07$ & $2.06 \mathrm{E}+07$ & 2.48 & 0.272 & 0.854 & No & No Sp & CYT \\
\hline & OKL & $6.18 \mathrm{E}+07$ & $1.23 \mathrm{E}+07$ & 5.05 & & & & & \\
\hline \multirow[t]{2}{*}{ PlpE } & $89010807 \mathrm{~N}$ & $5.87 \mathrm{E}+08$ & $2.91 \mathrm{E}+07$ & 20.17 & 0.68 & 0.928 & Yes & Sec Sp & SpII \\
\hline & OKL & $6.03 E+08$ & $1.49 \mathrm{E}+07$ & 40.47 & & & & & \\
\hline \multirow[t]{2}{*}{ OmpA } & $89010807 \mathrm{~N}$ & $2.30 \mathrm{E}+07$ & $7.45 \mathrm{E}+06$ & 3.08 & 0.918 & 0.950 & Yes & Sec Sp & SpI \\
\hline & OKL & $1.97 \mathrm{E}+07$ & $3.09 \mathrm{E}+06$ & 6.38 & & & & & \\
\hline \multirow[t]{2}{*}{ Serotype 1 Specific antigen } & $89010807 \mathrm{~N}$ & $1.98 \mathrm{E}+08$ & $6.47 \mathrm{E}+08$ & 0.31 & 0.733 & 0.914 & Yes & Sec Sp & SpI \\
\hline & OKL & $2.03 \mathrm{E}+08$ & $4.50 \mathrm{E}+08$ & 0.45 & & & & & \\
\hline \multirow[t]{2}{*}{ Omp P2 } & $89010807 \mathrm{~N}$ & $2.61 \mathrm{E}+06$ & ND & & 0.828 & 0.864 & Yes & Sec Sp & SpI \\
\hline & OKL & $2.20 \mathrm{E}+06$ & ND & & & & & & \\
\hline \multirow[t]{2}{*}{ GS60. } & $89010807 \mathrm{~N}$ & ND & $2.24 \mathrm{E}+06$ & & 0.532 & 0.114 & Yes & Sec Sp & SpII \\
\hline & OKL & ND & $1.24 \mathrm{E}+06$ & & & & & & \\
\hline \multirow[t]{2}{*}{ PlpF } & $89010807 \mathrm{~N}$ & $4.66 \mathrm{E}+08$ & $9.98 \mathrm{E}+07$ & 4.67 & 0.693 & 0.924 & Yes & Sec Sp & SpII \\
\hline & OKL & $3.85 \mathrm{E}+08$ & $5.92 \mathrm{E}+07$ & 6.51 & & & & & \\
\hline \multirow[t]{2}{*}{ TbpA } & $89010807 \mathrm{~N}$ & ND & $1.76 \mathrm{E}+08$ & & 0.788 & 0.897 & Yes & Sec Sp & SpI \\
\hline & OKL & ND & $1.59 \mathrm{E}+08$ & & & & & & \\
\hline \multirow[t]{2}{*}{ TbpB } & $89010807 \mathrm{~N}$ & $6.54 \mathrm{E}+07$ & $4.13 \mathrm{E}+08$ & 0.16 & 0.49 & 0.930 & Yes & Sec Sp & SpII \\
\hline & OKL & $4.12 \mathrm{E}+07$ & $2.01 \mathrm{E}+08$ & 0.20 & & & & & \\
\hline \multirow[t]{2}{*}{ IgA Protease } & $89010807 \mathrm{~N}$ & $5.78 \mathrm{E}+07$ & $6.75 \mathrm{E}+07$ & 0.86 & 0.666 & 0.956 & Yes & Sec Sp & SpI \\
\hline & OKL & $1.31 \mathrm{E}+08$ & $3.53 \mathrm{E}+07$ & 3.71 & & & & & \\
\hline \multirow[t]{2}{*}{ Omp P1 } & $89010807 \mathrm{~N}$ & $1.01 \mathrm{E}+07$ & $4.37 \mathrm{E}+06$ & 2.31 & 0.863 & 0.923 & Yes & Sec Sp & SpI \\
\hline & OKL & $7.95 \mathrm{E}+06$ & $3.46 \mathrm{E}+06$ & 2.30 & & & & & \\
\hline \multirow[t]{2}{*}{ Omp P5 } & $89010807 \mathrm{~N}$ & $1.27 \mathrm{E}+08$ & $5.06 \mathrm{E}+06$ & 25.10 & 0.918 & 0.950 & Yes & Sec Sp & SpI \\
\hline & OKL & $1.18 \mathrm{E}+08$ & $5.74 \mathrm{E}+06$ & 20.56 & & & & & \\
\hline \multirow[t]{2}{*}{ Omp P6 } & $89010807 \mathrm{~N}$ & $3.97 \mathrm{E}+06$ & $5.21 \mathrm{E}+06$ & 0.76 & 0.776 & 0.963 & Yes & Sec Sp & SpII \\
\hline & OKL & $3.57 \mathrm{E}+06$ & $3.90 \mathrm{E}+06$ & 0.92 & & & & & \\
\hline \multirow[t]{2}{*}{ Superoxide dismutase } & $89010807 \mathrm{~N}$ & $9.26 \mathrm{E}+07$ & $8.26 \mathrm{E}+07$ & 1.12 & 0.088 & 0.126 & No & No Sp & CYT \\
\hline & OKL & ND & $7.84 \mathrm{E}+07$ & & & & & & \\
\hline \multirow[t]{2}{*}{ BamA (Omp D15) } & $89010807 \mathrm{~N}$ & ND & $3.31 \mathrm{E}+06$ & & 0.744 & 0.843 & Yes & Sec Sp & SpI \\
\hline & OKL & $\mathrm{Nd}$ & $4.26 \mathrm{E}+06$ & & & & & & \\
\hline \multirow[t]{2}{*}{ Sialoglycoprotease } & $89010807 \mathrm{~N}$ & ND & $2.24 \mathrm{E}+06$ & & 0.671 & 0.898177 & Yes & Sec Sp & SpI \\
\hline & OKL & ND & $1.24 \mathrm{E}+06$ & & & & & & \\
\hline
\end{tabular}

SignalP4.1 score of $>0.51$ (0.57) - signal peptide present.

SecretomeP 2.0 of $>0.5$ - putative secreted protein.

Phobius - Yes $=$ signal peptide detected and No.

PRED-TAT - Sec Sp = sec signal peptide detected.

LipoP - CYT = cytoplasmic; SpI = substrate to Signal Peptidase I and SpII = substrate to Signal Peptidase II.

ND - Not detected. 
2010; Shrivastava and Miller, 2009), metabolic processes such as acquisition of nutrients (Bosch et al., 2002; Choi-Kim et al., 1991; Stojiljkovic and Hantke, 1992), adjusting to environmental pressures (Macdonald and Kuehn, 2013), potential candidates for vaccines (Choi et al., 2012; Mariappan et al., 2010; Naz et al., 2015; Song et al., 2013) or sometimes have been used as vaccines themselves (Shewen and Wilkie, 1988; Bottero et al., 2016).

$M$. haemolytica secretomes studied in this work contain outer membrane vesicles, proteins that are actively secreted and those that are released as a result of cytoplasmic leakage due to cell lysis. As the proteomic analysis of the secretome done in this work shows that it contains a long list of proteins that have already been shown to induce immunogenic responses that provide varying degrees of protection to challenge by isogenic strain of M. haemolytica.

$M$. haemolytica proteins whose immunogenicity have been established in ours and other laboratories include LKT (Gentry et al., 1985; Lo et al., 1987; Shewen and Wilkie, 1988), PlpE (Ayalew et al., 2004; Confer et al., 2003; Confer et al., 2006), PlpE-LKT chimera (Confer et al., 2009), serotype 1 specific antigen, OmpA, OmpD15, OmpP2 (Ayalew et al., 2011a,b), PlpF (Ayalew et al., 2011a, b), outer membrane vesicles (Ayalew et al., 2013), cholera toxin subunit B-PlpE-LKT chimera (Ayalew et al., 2009), sialoglycoprotease (Shewen et al., 2003), GS60 (Lee et al., 2008; Orouji et al., 2012), adhesins (Kisiela and Czuprynski, 2009; Klima et al., 2014), and iron-regulated OMPs (Deneer and Potter, 1989; Potter et al., 1999). Representative M. haemolytica proteins whose immunogenicity has been shown in our earlier works and other investigators are given in Table 7. The table provides information on whether each protein is detected in secretomes from $M$. haemolytica strains $89010807 \mathrm{~N}$ and OKL, as well as their relative abundance and fold change as approximated by LFQ intensity and predictions by four bioinformatic tools. The complete list of proteins identified in $M$. haemolytica secretome is provided in the Supplementary Table. The LC-MS/MS intensities of its individual peptides and not exact quantification of each protein nor the sum of the intensities of each peptide, rather it is a complex algebraic solution to all of the peptide intensities in the set of samples and it represents normalized protein abundance. The M. haemolytica secretome characterized in this study contains all proteins whose recombinant forms have been shown to induce varying degrees of immune response, which may be partially protective individually, or in combination. The fact that a subset of this secretome (outer membrane vesicles) has been shown to be immunogenic not only in M. haemolytica but other pathogenic bacteria, and the fact that production of secretomes is not as time consuming and costly as producing individual recombinant proteins (Ayalew et al., 2013) would lead us to conclude this research has identified a substantial number of proteins in secretomes of Mannheimia haemolytica that may induce protective immune response, making such preparations attractive multivalent vaccine candidates.

\section{Acknowledgements}

This project was supported in part by Agriculture and Food Research Initiative Competitive Grant no. 2009-01626 from the USDA National Institute of Food and Agriculture.

\section{References}

Ayalew, S., Confer, A.W., Blackwood, E.R., 2004. Characterization of immunodominant and potentially protective epitopes of Mannheimia haemolytica serotype 1 outer membrane lipoprotein PlpE. Infect. Immun. 72, 7265-7274.

Ayalew, S., Confer, A.W., Hartson, S.D., Shrestha, B., 2010. Immunoproteomic analyses of outer membrane proteins of Mannheimia haemolytica and identification of potential vaccine candidates. Proteomics 10, 2151-2164.

Ayalew, S., Confer, A.W., Shrestha, B., Wilson, A.E., Montelongo, M., 2013. Proteomic analysis and immunogenicity of Mannheimia haemolytica vesicles. Clin. Vaccine Immunol. 20, 191-196.

Ayalew, S., Shrestha, B., Montelongo, M., Wilson, A.E., Confer, A.W., 2011a. Immunogenicity of Mannheimia haemolytica recombinant outer membrane proteins serotype 1 -specific antigen, OmpA, OmpP2, and OmpD15. Clin. Vaccine Immunol. 18, 2067-2074.

Ayalew, S., Shrestha, B., Montelongo, M., Wilson, A., Confer, A.W., 2011b. Identification and immunogenicity of Mannheimia haemolytica S1 outer membrane lipoprotein PlpF. Vaccine 39 (47), 8712-8718.

Ayalew, S., Step, D.L., Montelongo, M., Confer, A.W., 2009. Intranasal vaccination of calves with Mannheimia haemolytica chimeric protein containing the major surface epitope of outer membrane lipoprotein PlpE, the neutralizing epitope of leukotoxin, and cholera toxin subunit B. Vet. Immunol. Immunopathol. 132, 295-302.

Bagos, P.G., Nikolaou, E.P., Liakopoulos, T.D., Tsirigos, K.D., 2010. Combined prediction of Tat and Sec signal peptides with hidden Markov models. Bioinformatics 26, 2811-2817.

Bendtsen, J.D., Kiemer, L., Fausboll, A., Brunak, S., 2005a. Non-classical protein secretion in bacteria. BMC Microbiol. 5, 58.

Bendtsen, J.D., Nielsen, H., Widdick, D., Palmer, T., Brunak, S., 2005b. Prediction of twin-arginine signal peptides. BMC Bioinf. 6, 167.

Bosch, M., Garrido, M.E., Llagostera, M., Perez de Rozas, A.M., Badiola, I., Barbe, J., 2002. Characterization of the Pasteurella multocida hgbA gene encoding a hemoglobin-binding protein. Infect. Immun. 70, 5955-5964.

Bottero, D., Gaillard, M.E., Zurita, E., Moreno, G., Martinez, D.S., Bartel, E., Bravo, S., Carriquiriborde, F., Errea, A., Castuma, C., Rumbo, M., Hozbor, D., 2016. Characterization of the immune response induced by pertussis OMVs-based vaccine. Vaccine .

Camacho, C., Coulouris, G., Avagyan, V., Ma, N., Papadopoulos, J., Bealer, K., Madden, T.L., 2009. BLAST+: architecture and applications. BMC Bioinf. 10, 421.

Chang, A., Schomburg, I., Placzek, S., Jeske, L., Ulbrich, M., Xiao, M., Sensen, C.W., Schomburg, D., 2015. BRENDA in 2015: exciting developments in its 25th year of existence. Nucleic Acids Res. 43, D439-D446.

Choi-Kim, K., Maheswaran, S.K., Felice, L.J., Molitor, T.W., 1991. Relationship between the iron regulated outer membrane proteins and the outer membrane proteins of in vivo grown Pasteurella multocida. Vet. Microbiol. 28, 75-92.

Choi, C.W., Lee, Y.G., Kwon, S.O., Kim, H.Y., Lee, J.C., Chung, Y.H., Yun, C.Y., Kim, S.I., 2012. Analysis of Streptococcus pneumoniae secreted antigens by immunoproteomic approach. Diagn. Microbiol. Infect. Dis. 72, 318-327.

Confer, A.W., 1993. Immunogens of pasteurella. Vet. Microbiol. 37, 353-368.

Confer, A.W., Ayalew, S., Montelongo, M., Step, D.L., Wray, J.H., Hansen, R.D., Panciera, R.J., 2009. Immunity of cattle following vaccination with a Mannheimia haemolytica chimeric PlpE-LKT (SAC89) protein. Vaccine 27, 17711776.

Confer, A.W., Ayalew, S., Panciera, R.J., Montelongo, M., Whitworth, L.C., Hammer, J. D., 2003. Immunogenicity of recombinant Mannheimia haemolytica serotype 1 outer membrane protein PlpE and augmentation of a commercial vaccine. Vaccine 21, 2821-2829.

Confer, A.W., Ayalew, S., Panciera, R.J., Montelongo, M., Wray, J.H., 2006. Recombinant Mannheimia haemolytica serotype 1 outer membrane protein PlpE enhances commercial $M$. haemolytica vaccine-induced resistance against serotype 6 challenge. Vaccine 24, 2248-2255.

Costa, T.R., Felisberto-Rodrigues, C., Meir, A., Prevost, M.S., Redzej, A., Trokter, M., Waksman, G., 2015. Secretion systems in Gram-negative bacteria: structural and mechanistic insights. Nat. Rev. Microbiol. 13, 343-359.

Cox, J., Hein, M.Y., Luber, C.A., Paron, I., Nagaraj, N., Mann, M., 2014. Accurate proteome-wide label-free quantification by delayed normalization and maximal peptide ratio extraction, termed MaxLFQ. Mol. Cell. Proteomics 13, 2513-2526.

Cox, J., Mann, M., 2008. MaxQuant enables high peptide identification rates, individualized p.p.b.-range mass accuracies and proteome-wide protein quantification. Nat. Biotechnol. 26, 1367-1372.

Deneer, H.G., Potter, A.A., 1989. Iron-repressible outer-membrane proteins of Pasteurella haemolytica. J. Gen. Microbiol. 135 (Pt 2), 435-443.

Eddy, S.R., 2011. Accelerated profile HMM searches. PLoS Comput. Biol. 7, e1002195.

Finn, R.D., Bateman, A., Clements, J., Coggill, P., Eberhardt, R.Y., Eddy, S.R., Heger, A., Hetherington, K., Holm, L., Mistry, J., Sonnhammer, E.L., Tate, J., Punta, M., 2014. Pfam: the protein families database. Nucleic Acids Res. 42, D222-D230.

Finn, R.D., Clements, J., Arndt, W., Miller, B.L., Wheeler, T.J., Schreiber, F., Bateman, A., Eddy, S.R., 2015. HMMER web server: 2015 update. Nucleic Acids Res. 43, W30W38.

Frank, G.H., 1986. The role of Pasteurella haemolytica in the bovine respiratory disease complex. Vet. Med. 81, 838-846.

Frank, G.H., 1989. Pasteurellosis of Cattle. Academic Press Limited, London, pp. 197222 
Gagic, D., Ciric, M., Wen, W.X., Ng, F., Rakonjac, J., 2016. Exploring the secretomes of microbes and microbial communities using filamentous phage display. Front. Microbiol. 7, 429.

Galperin, M.Y., Makarova, K.S., Wolf, Y.I., Koonin, E.V., 2015. Expanded microbial genome coverage and improved protein family annotation in the COG database. Nucleic Acids Res. 43, D261-D269.

Gentry, M.J., Confer, A.W., Panciera, R.J., 1985. Serum neutralization of cytotoxin from Pasteurella haemolytica, serotype 1 and resistance to experimental bovine pneumonic pasteurellosis. Vet. Immunol. Immunopathol. 9, 239-250.

Green, E.R., Mecsas, J., 2016. Bacterial secretion systems: an overview. Microbiol. Spectr. 4.

Juncker, A.S., Willenbrock, H., Von Heijne, G., Brunak, S., Nielsen, H., Krogh, A., 2003. Prediction of lipoprotein signal peptides in Gram-negative bacteria. Protein Sci. $12,1652-1662$

Kall, L., Krogh, A., Sonnhammer, E.L., 2007. Advantages of combined transmembrane topology and signal peptide prediction - the Phobius web server. Nucleic Acids Res. 35, W429-W432.

Kisiela, D.I., Czuprynski, C.J., 2009. Identification of Mannheimia haemolytica adhesins involved in binding to bovine bronchial epithelial cells. Infect. Immun. 77, 446-455.

Klima, C.L., Alexander, T.W., Hendrick, S., McAllister, T.A., 2014. Characterization of Mannheimia haemolytica isolated from feedlot cattle that were healthy or treated for bovine respiratory disease. Can. J. Vet. Res. 78, 38-45.

Konecna, K., Hernychova, L., Reichelova, M., Lenco, J., Klimentova, J., Stulik, J., Macela, A., Alefantis, T., Delvecchio, V.G., 2010. Comparative proteomic profiling of culture filtrate proteins of less and highly virulent Francisella tularensis strains. Proteomics 10, 4501-4511.

Kuehn, M.J., Kesty, N.C., 2005. Bacterial outer membrane vesicles and the hostpathogen interaction. Genes Dev. 19, 2645-2655.

Larson, R.L., Step, D.L., 2012. Evidence-based effectiveness of vaccination against Mannheimia haemolytica, Pasteurella multocida, and Histophilus somni in feedlot cattle for mitigating the incidence and effect of bovine respiratory disease complex. Vet. Clin. North Am. Food Anim. Pract. 28, 97-106 106e101-107, ix.

Lee, C.W., Shewen, P.E., Cladman, W.M., Conlon, J.A., Mellors, A., Lo, R.Y., 1994. Sialoglycoprotease of Pasteurella haemolytica A1: detection of antisialoglycoprotease antibodies in sera of calves. Can. J. Vet. Res. 58, 93-98.

Lee, R.W., Cornelisse, M., Ziauddin, A., Slack, P.J., Hodgins, D.C., Strommer, J.N., Shewen, P.E., Lo, R.Y., 2008. Expression of a modified Mannheimia haemolytica GS60 outer membrane lipoprotein in transgenic alfalfa for the development of an edible vaccine against bovine pneumonic pasteurellosis. J. Biotechnol. 135, 224-231.

Lo, R.Y., Strathdee, C.A., Shewen, P.E., 1987. Nucleotide sequence of the leukotoxin genes of Pasteurella haemolytica A1. Infect. Immun. 55, 1987-1996.

Lycklama, A.N.J.A., Driessen, A.J., 2012. The bacterial Sec-translocase: structure and mechanism. Philos. Trans. R. Soc. Lond. B: Biol. Sci. 367, 1016-1028.

Macdonald, I.A., Kuehn, M.J., 2013. Stress-induced outer membrane vesicle production by Pseudomonas aeruginosa. J. Bacteriol. 195, 2971-2981.

Mariappan, V., Vellasamy, K.M., Thimma, J.S., Hashim, O.H., Vadivelu, J., 2010. Identification of immunogenic proteins from Burkholderia cepacia secretome using proteomic analysis. Vaccine 28, 1318-1324.

Miles, D.G., 2009. Overview of the North American beef cattle industry and the incidence of bovine respiratory disease (BRD). Anim. Health Res. Rev. 10, 101 103.

Morton, R.J., Panciera, R.J., Fulton, R.W., Frank, G.H., Ewing, S.A., Homer, J.T., Confer, A.W., 1995. Vaccination of cattle with outer membrane protein-enriched fractions of Pasteurella haemolytica and resistance against experimental challenge exposure. Am. J. Vet. Res. 56, 875-879.
Mosier, D.A., Simons, K.R., Chengappa, M.M., Confer, A.W., 1994. Antigenic composition of Pasteurella haemolytica serotype-1 supernatants from supplemented and nonsupplemented media. Am. J. Vet. Res. 55, 348-352.

Mosier, D.A., Simons, K.R., Confer, A.W., Panciera, R.J., Clinkenbeard, K.D., 1989. Pasteurella haemolytica antigens associated with resistance to pneumonic pasteurellosis. Infect. Immun. 57, 711-716.

Naz, A., Awan, F.M., Obaid, A., Muhammad, S.A., Paracha, R.Z., Ahmad, J., Ali, A., 2015. Identification of putative vaccine candidates against Helicobacter pylori exploiting exoproteome and secretome: a reverse vaccinology based approach. Infect. Genet. Evol. 32, 280-291.

Orouji, S., Hodgins, D.C., Lo, R.Y., Shewen, P.E., 2012. Serum IgG response in calves to the putative pneumonic virulence factor Gs60 of Mannheimia haemolytica A1. Can. J. Vet. Res. 76, 292-300.

Palmer, T., Berks, B.C., 2012. The twin-arginine translocation (Tat) protein export pathway. Nat. Rev. Microbiol. 10, 483-496.

Pandher, K., Murphy, G.L., Confer, A.W., 1999. Identification of immunogenic, surface-exposed outer membrane proteins of Pasteurella haemolytica serotype 1. Vet. Microbiol. 65, 215-226.

Perino, L.J., Hunsaker, B.D., 1997. A review of bovine respiratory disease vaccine field efficacy. Bovine Pract. 31, 59-66.

Petersen, T.N., Brunak, S., von Heijne, G., Nielsen, H., 2011. SignalP 4.0: discriminating signal peptides from transmembrane regions. Nat. Methods 8 , 785-786.

Potter, A.A., Schryvers, A.B., Ogunnariwo, J.A., Hutchins, W.A., Lo, R.Y., Watts, T., 1999. Protective capacity of the Pasteurella haemolytica transferrin-binding proteins TbpA and TbpB in cattle. Microb. Pathog. 27, 197-206.

Rice, J.A., Carrasco-Medina, L., Hodgins, D.C., Shewen, P.E., 2007. Mannheimia haemolytica and bovine respiratory disease. Anim. Health Res. Rev. 8, 117-128.

Rodriguez-Alvarez, M., Palomec-Nava, I.D., Mendoza-Hernandez, G., Lopez-Vidal, Y. 2010. The secretome of a recombinant BCG substrain reveals differences in hypothetical proteins. Vaccine 28, 3997-4001.

Romine, M.F., 2011. Genome-wide protein localization prediction strategies for gram negative bacteria. BMC Genomics 12 (Suppl. 1), S1.

Shewen, P.E., Lee, C.W., Perets, A., Hodgins, D.C., Baldwin, K., Lo, R.Y., 2003. Efficacy of recombinant sialoglycoprotease in protection of cattle against pneumonic challenge with Mannheimia (Pasteurella) haemolytica A1. Vaccine 21,1901-1906.

Shewen, P.E., Wilkie, B.N., 1988. Vaccination of calves with leukotoxic culture supernatant from Pasteurella haemolytica. Can. J. Vet. Res. 52, 30-36.

Shrivastava, R., Miller, J.F., 2009. Virulence factor secretion and translocation by Bordetella species. Curr. Opin. Microbiol. 12, 88-93.

Song, M., Xie, J., Peng, X., Li, H., 2013. Identification of protective immunogens from extracellular secretome of Edwardsiella tarda. Fish Shellfish Immunol. 35, 1932 1936.

Stojiljkovic, I., Hantke, K., 1992. Hemin uptake system of Yersinia enterocolitica: similarities with other TonB-dependent systems in gram-negative bacteria. EMBO J. 11, 4359-4367.

Tatusov, R.L., Koonin, E.V., Lipman, D.J., 1997. A genomic perspective on protein families. Science 278, 631-637.

UniProt, C., 2015. UniProt: a hub for protein information. Nucleic Acids Res. 43, D204-D212.

Voruganti, S., Lacroix, J.C., Rogers, C.N., Rogers, J., Matts, R.L., Hartson, S.D., 2013. The anticancer drug AUY922 generates a proteomics fingerprint that is highly conserved among structurally diverse Hsp90 inhibitors. J. Proteome Res. 12, 3697-3706.

Yu, N.Y., Wagner, J.R., Laird, M.R., Melli, G., Rey, S., Lo, R., Dao, P., Sahinalp, S.C., Ester, M., Foster, L.J., Brinkman, F.S., 2010. PSORTb 3.0: improved protein subcellular localization prediction with refined localization subcategories and predictive capabilities for all prokaryotes. Bioinformatics 26, 1608-1615. 